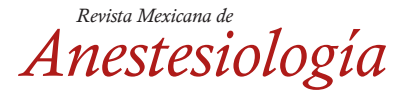

Enero-Marzo 2022

Vol. 45. No. 1. pp 48-59

doi: $10.35366 / 102903$

\section{Tromboelastografía para cirugía general}

\author{
Thromboelastography for general surgery
}

\author{
Dra. Ana Luisa Hernández-Pérez,* Dra. Karina Ramírez-Morales,* \\ Dr. Juan Lagarda-Cuevas, ${ }^{*}$ Dra. Cristina Revilla-Monsalve, ${ }^{\ddagger}$ \\ Dr. Gerardo Manuel Bermúdez-Ochoa,* \\ Dr. José Salvador Juárez-Pichardo,* Dra. Ana Gabriela Gallardo-Hernández ${ }^{\ddagger}$
}

Citar como: Hernández-Pérez AL, Ramírez-Morales K, Lagarda-Cuevas J, Revilla-Monsalve C, Bermúdez-Ochoa GM, Juárez-Pichardo JS, et al. Tromboelastografía para cirugía general. Rev Mex Anestesiol. 2022; 45 (1): 48-59. https:// dx.doi.org/10.35366/102903

RESUMEN. Las pruebas convencionales de coagulación evalúan el tiempo en que los factores de coagulación se activan en el plasma sanguíneo, el cual carece de componentes celulares, como enzimas y plaquetas que intervienen en el desarrollo de la hemostasia. La tromboelastografía (TEG) realiza un análisis in vitro de la relación entre plaquetas, enzimas, fibrinógeno y otros elementos de la coagulación de manera integral; por lo que se utiliza con mayor frecuencia en cirugías cardíacas, trasplantes y cirugías de malformaciones arteriovenosas, donde la pérdida sanguínea esperada es mayor a $40 \%$ del volumen sanguíneo circulante. La inclusión de la TEG en la valoración preanestésica permite evaluar la actividad plaquetaria en los pacientes que utilizan antiagregantes, como clopidogrel o ácido acetilsalicílico, a través del mapeo plaquetario (Platelet Mapping ${ }^{\circledR}$ ). Cuando se presenta hemorragia en el período transanestésico, la TEG permite identificar de manera específica el tipo de tratamiento necesario para mejorar la coagulación o la transfusión de elementos formes de la sangre. La TEG permite realizar protocolos más sofisticados de terapia transfusional, lo que implica una disminución de las complicaciones asociadas con la politransfusión y, por ende, la disminución de costos, por lo que estos beneficios justifican el uso rutinario de la TEG para cualquier cirugía general.

ABSTRACT. Conventional coagulation tests evaluate the time in which the coagulation factors are activated in the blood plasma, which lacks cellular components such as enzymes and platelets that are involved in the development of hemostasis. Thromboelastography (TEG) performs an in vitro analysis of the relationship between platelets, enzymes, fibrinogen, and other coagulation elements in an integral way; reason why it is used more frequently in cardiac surgeries, transplants, and surgeries of arteriovenous malformations, where the expected blood loss is greater than $40 \%$ of the circulating blood volume. The inclusion of TEG in the pre-anesthetic evaluation allows evaluating platelet activity in patients who use antiplatelets therapy, such as clopidogrel or acetylsalicylic acid, through platelet mapping (Platelet Mapping ${ }^{\circledR}$ ). When bleeding occurs in the trans-anesthetic period, TEG specifically identifies the type of treatment necessary to improve coagulation or transfusion of formed blood elements. The TEG allows more sophisticated transfusion therapy protocols to be carried out, which implies a decrease in complications associated with polytransfusion and a reduction in costs, so these benefits justify the routine use of TEG for any general surgery.

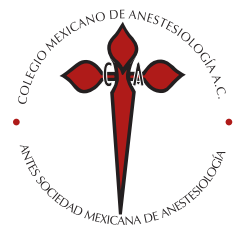

Palabras clave: Tromboelastografía, coagulación, mapeo plaquetario.

Keywords:

Thromboelastography, coagulation, platelet mapping.

* Centro Médico ABC.

* Unidad de Investigación Médica en Enfermedades Metabólicas, IMSS.

Correspondencia:

Dra. Ana Gabriela Gallardo-Hernández Unidad de Investigación en Enfermedades Metabólicas, Instituto Mexicano del Seguro Social. E-mail: anagabygh@gmail.com

Recibido: 07-05-2020 Aceptado: 02-10-2021

\section{INTRODUCCIÓN}

I a hemostasia es un mecanismo de defensa del organismo, $\_$necesario para mantener la integridad de la pared vascular, evitar la pérdida de sangre ante una lesión vascular y reestablecer el flujo sanguíneo cuando se ha reparado la lesión, sobre todo cuando un paciente es sometido a cualquier tipo de cirugía. Más de $60 \%$ de las transfusiones sanguíneas son efectuadas con relación directa al acto quirúrgico, lo que aumenta el riesgo de complicaciones que pueden evolucionar a un estado crítico del paciente, como reacciones alérgicas, síndrome de estrés respiratorio, insuficiencia cardíaca y en ocasiones el riesgo de contraer infecciones virales como VIH o hepatitis ${ }^{(1)}$. Las pruebas prequirúrgicas convencionales examinan la coagulación por medio de un análisis independiente de la vía intrínseca y extrínseca de la cascada de coagulación mediante un proceso donde sólo es empleado el plasma sanguíneo. El mecanismo por el cual la coagulación permite la hemostasia es un proceso complejo que se realiza a través de una serie de factores de coagulación ${ }^{(2)}$.

La vía intrínseca consta de los factores I, II, IX, X, XI y XII. La denominación de cada uno de los factores se muestra en la Tabla 1. Todos los factores de coagulación al ser activados se les denominan con un «a», después del número del factor. Un factor ya activado funciona como catalizador para activar el siguiente factor de la cascada.

La vía intrínseca es la más larga de la hemostasia secundaria. Comienza con la activación del factor XII, que se convierte en factor XIIa después de la exposición al colágeno endotelial, al cual sólo se expone cuando se produce daño endotelial. El 
factor XIIa activa el factor XI en factor XIa, éste al factor IX en factor IXa. El factor IXa es un catalizador para el factor $\mathrm{X}$ que lo convierte en factor Xa; esta reacción en cadena es la que se conoce como cascada de coagulación de la vía intrínseca. La vía intrínseca se mide clínicamente como el tiempo parcial de tromboplastina (TTP) ${ }^{(2,3)}$.

A medida que avanza en la cascada, la concentración de cada factor activado aumenta en sangre. Por ejemplo, la concentración del factor IX es mayor que la del factor XI. Cuando el factor II se activa por vía intrínseca o extrínseca, puede reforzar la vía intrínseca al dar retroalimentación positiva a los factores V, VII, VIII, XI, XIII. Esto hace que el factor XII sea menos indispensable y la sangre pueda coagularse bien sin factor $\mathrm{XII}^{(2)}$.

La vía extrínseca consta de los factores I, II, VII y X. La denominación de los factores se muestra en la Tabla 1. Es la vía más corta de la hemostasia secundaria, una vez que se produce daño a las células endoteliales, éstas liberan factor tisular que activa el factor VII en factor VIIa. El factor VIIa continúa para activar el factor $\mathrm{X}$ en el factor Xa. Este es el punto donde las vías extrínsecas e intrínsecas se vuelven una; la vía extrínseca se mide clínicamente como el tiempo de protrombina (TP) ${ }^{(2,3)}$.

La vía común consiste en factores I, II, V, VIII, X. Estos factores circulan a través del torrente sanguíneo como zimógenos y se activan en serina proteasas, las cuales actúan como un catalizador para separar el siguiente zimógeno en más serina proteasas y finalmente activar el fibrinógeno, que es el factor $\mathrm{I}^{(3,4)}$.

La vía intrínseca se activa a través del colágeno endotelial expuesto, y la vía extrínseca se activa a través del factor tisular liberado por las células endoteliales después de un daño externo ${ }^{(3-5)}$.

También participan componentes celulares como las enzimas y principalmente las plaquetas que intervienen en el desarrollo de la hemostasia y que al no estar presentes en el plasma manifiestan la carencia de información que brindan las pruebas de laboratorio que sólo analizan el tiempo en que los factores de coagulación son activados, comparando este análisis con la tromboelastografía podemos observar que el análisis de una muestra de sangre completa representa un monitoreo ideal para la hemostasia.

\section{Fases de la coagulación}

Primera fase o hemostasia primaria vascular plaquetaria. Es el período en que participan las plaquetas mediante las siguientes etapas:

1. Adhesión de plaquetas que se unen al colágeno del subendotelio vascular mediante receptores de membrana.

2. Activación de plaquetas la cual se realiza a través de la síntesis de tromboxano A3 y prostaglandinas.

3. Agregación de plaquetas con ayuda del factor de Von Willebrand y adenosín difosfato (ADP), formando así el tapón hemostático primario(6).

Segunda fase plasmática o de amplificación. La fase de amplificación es dependiente de la presencia de membranas plaquetarias activadas y de la interacción de éstas con los

\begin{tabular}{|c|c|c|}
\hline Número de factor & Nombre del factor & Cualidad \\
\hline I & Fibrinógeno & \\
\hline II & Protrombina & $\begin{array}{l}\text { Serina proteasa* } \\
\text { Dependiente de vitamina } K\end{array}$ \\
\hline III & Factor tisular & Cofactor ${ }^{\star *}$ \\
\hline IV & Calcio & Cofactor** \\
\hline V & Proacelerina & \\
\hline VII & Factor estable & $\begin{array}{l}\text { Serina proteasa* } \\
\text { Dependiente de vitamina } \mathrm{K}\end{array}$ \\
\hline VIII & Factor antihemofílico A & Cofactor** \\
\hline IX & Factor Christmas o antihemofílico B & $\begin{array}{l}\text { Serina proteasa* } \\
\text { Dependiente de vitamina } \mathrm{K}\end{array}$ \\
\hline$x$ & Factor Stuart Prower & $\begin{array}{l}\text { Autoprotrombina } \\
\text { Serina proteasa* } \\
\text { Dependiente de vitamina K }\end{array}$ \\
\hline XI & Antecedente tromboplastínico del plasma $\mathrm{o}$ antihemofílico $\mathrm{C}$ & Serina proteasa* \\
\hline XII & Factor de Hageman & Serina proteasa* \\
\hline XIII & Factor estabilizador de la fibrina & \\
\hline Precalicreína & Factor de Fletcher & \\
\hline Trombomodulina & & Cofactor** \\
\hline
\end{tabular}

* Enzimas que hidrolizan las proteínas transformándolas en aminoácidos o en péptidos sencillos.

** Tienen actividad catalítica, aceleran la velocidad de reacción. 


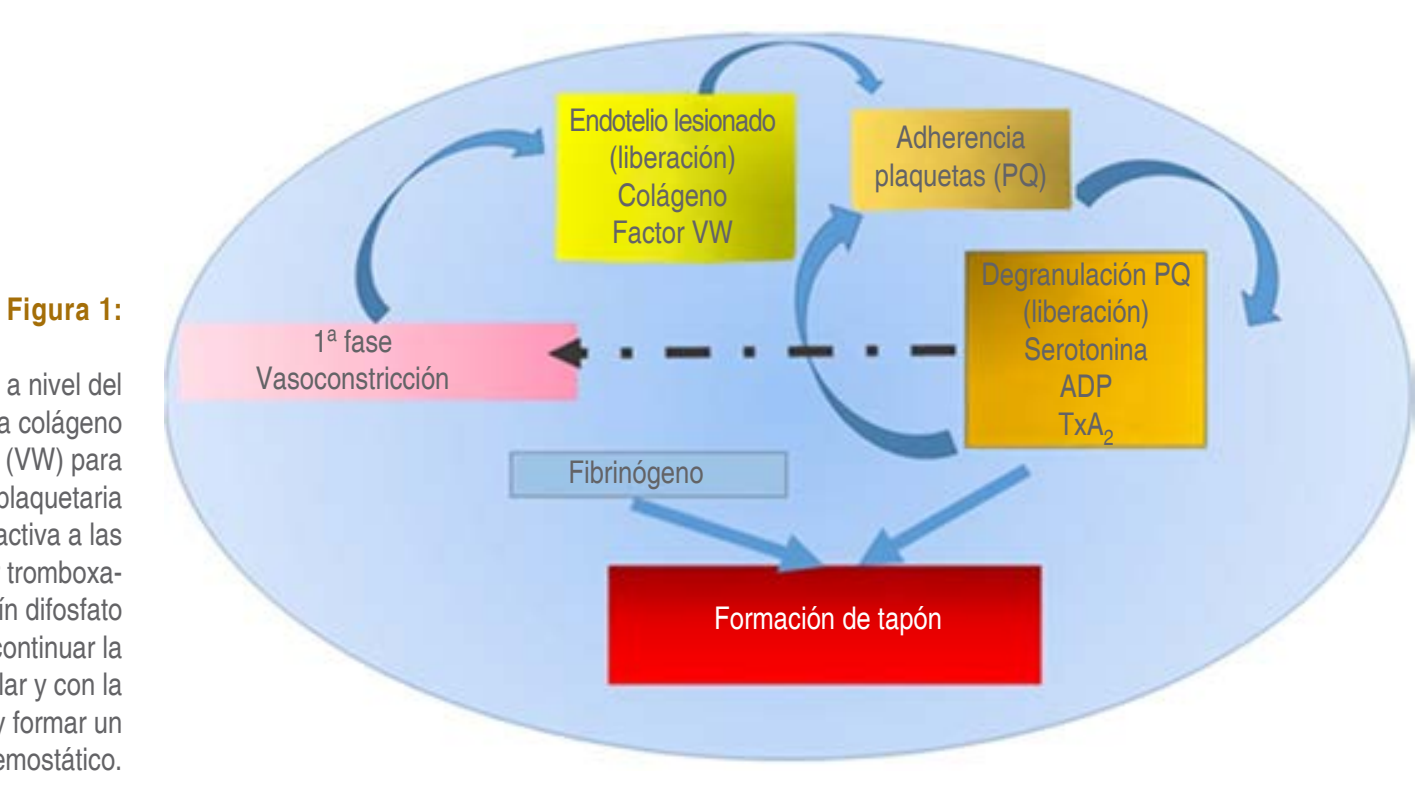

Ante la lesión del vaso, a nivel del endotelio vascular se libera colágeno y factor de Von Willebrand (VW) para que ayuden a la adhesión plaquetaria

al subendotelio, lo que activa a las plaquetas para liberar tromboxano A2 (TxA2), adenosín difosfato (ADP) y serotonina para continuar la vasoconstricción vascular y con la agregación plaquetaria y formar un tapón hemostático.

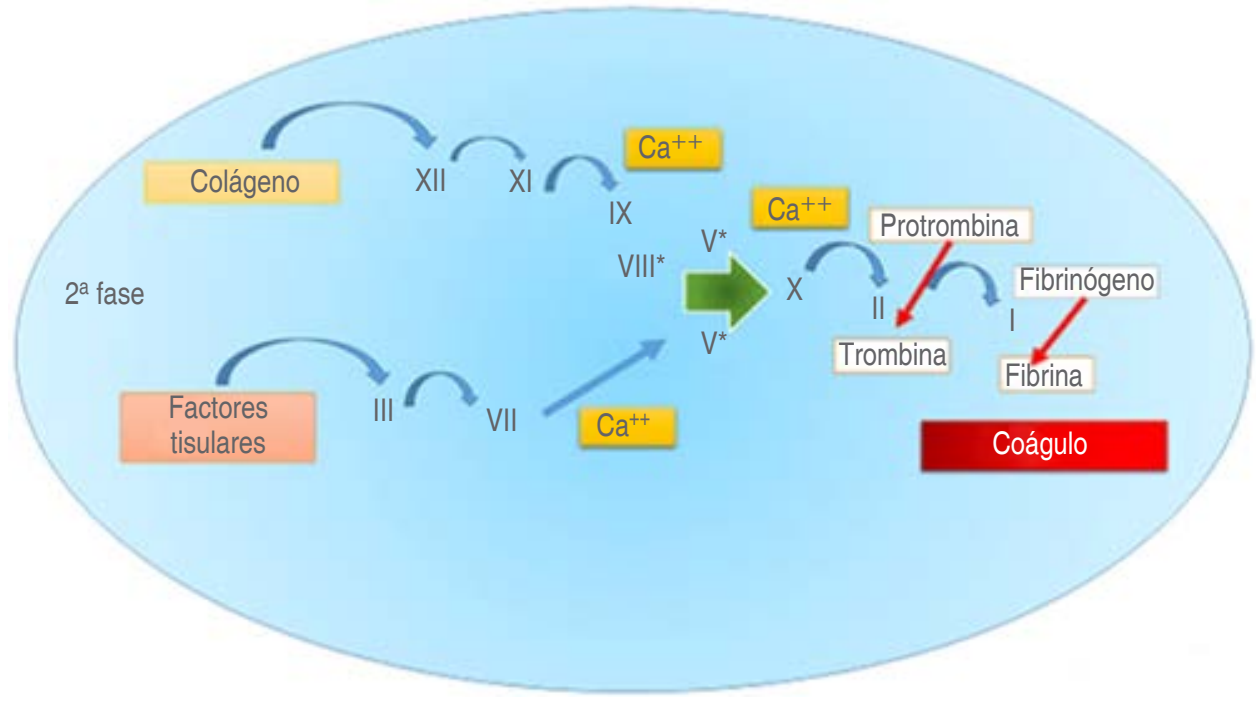

Figura 2:

Representación gráfica de la segunda fase de la coagulación que es la formación del coágulo a través de las vías extrínseca e intrínseca de la coagulación. Las flechas curvas indican la activación de los factores. ${ }^{*}$ Factores V y VIII se activan solos.

factores de coagulación. Se forma la trombina (factor II), junto con el calcio (factor IV) y los fosfolípidos (que provienen de la plaqueta), participan activamente en un proceso de retroalimentación para la activación de los factores XI, IX, VIII, V y para acelerar la activación de las plaquetas ${ }^{(6,7)}$.

Esta fase también se caracteriza por la activación del sistema de retroalimentación negativa a través de los anticoagulantes naturales TFPI (inhibidor de la activación del complejo FT/FVIIa), antitrombina y proteína $\mathrm{C}$, su función es regular los procesos procoagulantes (Figuras 1 y 2) ${ }^{(6,7)}$.

Tercera fase trombodinámica. En esta última fase de la coagulación se produce la destrucción del coágulo. La coagulación está regulada por anticoagulantes naturales: la proteína
C y la proteína S (que actúa como cofactor) que inactivan el FVIII y FV; la antitrombina, que inhibe principalmente al FXa y la trombina y, en menor grado, los factores IXa, XIa y XIIa y el inhibidor del factor tisular. En último lugar, la restauración de la permeabilidad vascular se establece por la fibrinólisis: la plasmina, proteína final de la vía fibrinolítica, degrada la fibrina, apareciendo los productos de degradación de la fibrina y los dímeros-D. La generación de la plasmina desde el plasminógeno está estimulada por el activador tisular del plasminógeno y la reacción está limitada por el inhibidor del activador del plasminógeno (IAP), por la a2-antiplasmina y por el inhibidor de la fibrinólisis activable por trombina (a través del complejo trombina-trombomodulina), que actúan a nivel distal ${ }^{(8)}$. 


\section{Pruebas de coagulación}

Las pruebas de coagulación se usan comúnmente para identificar defectos de las vías comunes extrínsecas, intrínsecas y finales de la cascada de coagulación para que se puedan realizar pruebas más avanzadas para identificar defectos específicos. Las mediciones de TS, TP, TTPa, TT y fibrinógeno se usan para detectar anormalidades de la hemostasia secundaria. La medición del fibrinógeno detecta deficiencias y defectos en la actividad del fibrinógeno plasmático $^{(9,10)}$.

\section{Tiempo de sangrado (TS)}

El tiempo de sangrado detecta alteraciones en las plaquetas. Se lleva a cabo mediante la realización de una pequeña punción en la oreja, se puede realizar la técnica de Duke o de Ivy. Se evalúa la ausencia del factor de Von Willebrand, uso de medicamentos anticoagulantes o trombocitopenia.
En caso de que se realice la técnica de Ivy, el tiempo normal de sangrado es de 6 a 9 minutos. En la técnica de Duke, el tiempo normal de sangrado es de 1 a 3 minutos ${ }^{(9-11)}$.

\section{Tiempo de protrombina (TP)}

La protrombina (factor II) tiene la función de promover la conversión del fibrinógeno en fibrina, formando el tapón plaquetario secundario o definitivo.

Este examen evalúa la vía extrínseca de la coagulación, puesto que determina el tiempo que la sangre tarda para formar el tapón secundario después de la exposición a la tromboplastina cálcica, que es el reactivo usado en la prueba.

En condiciones normales, luego del contacto de la sangre con la tromboplastina cálcica, la vía extrínseca se activa, produciendo los factores VII y X de la coagulación, por consecuencia, el factor II, que es la protrombina, promueve la conversión del fibrinógeno en fibrina, deteniendo el sangrado. Este proceso normalmente dura entre 10 a 14 segundos ${ }^{(1,11,12)}$.

\section{Elementos del tromboelastógrafo}

Figura 3:

En la copa se introduce $0.36 \mathrm{~mL}$ de muestra sanguínea, se establece la temperatura del paciente, el eje oscilante se empieza a mover de 4 a $75^{\circ}$, y el sensor del pin evalúa la viscosidad de la formación del coágulo, transfiriendo la información a través de un detector y después pasando la información a un procesador de datos, el cual nos da una imagen de la tromboelastografía en $15 \mathrm{~min}$.

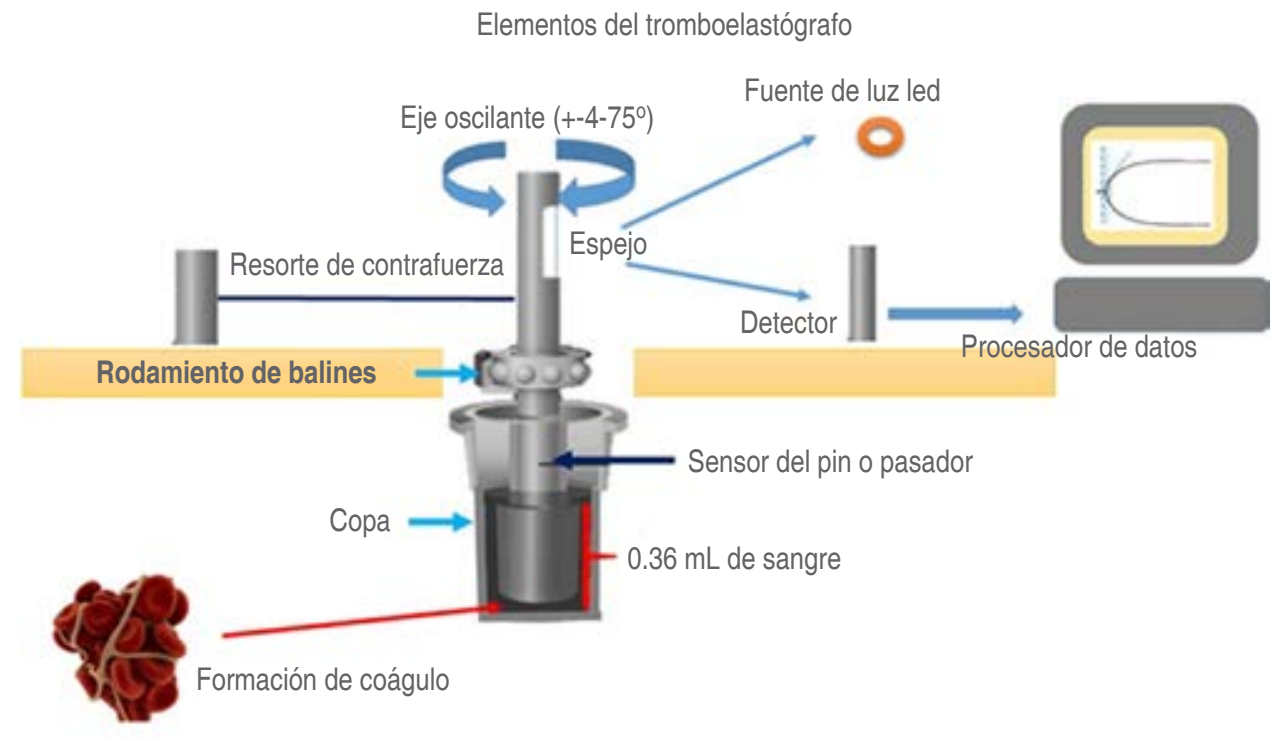

Figura 4:

Representación de tromboelastografía a los 45 min de iniciado el coágulo. Donde aparecen los valores de la muestra de cada uno de los parámetros. 
Figura 5:

En la tromboelastografía el parámetro $\mathrm{R}$ representa el inicio de la coagulación, donde la vía extrínseca inicia con la activación del factor II 0 tisular junto con el factor VII; la vía intrínseca comienza a través de la acción de la calicreína más el factor $X I I$. Ambas vías activan al factor $X$ de la vía común. Los parámetros $\mathrm{K}$ y ángulo alfa simbolizan la vía común de la cascada de coagulación y en asociación al factor IV (calcio)

y factor $V$, ayudan a la formación de protrombinasa. La MA (máxima amplitud) mide la fortaleza del coágulo, el cual se forma a través de los monómeros de fibrina con ayuda del factor XII y calcio. El parámetro LY30 mide el tiempo de fibrinólisis.

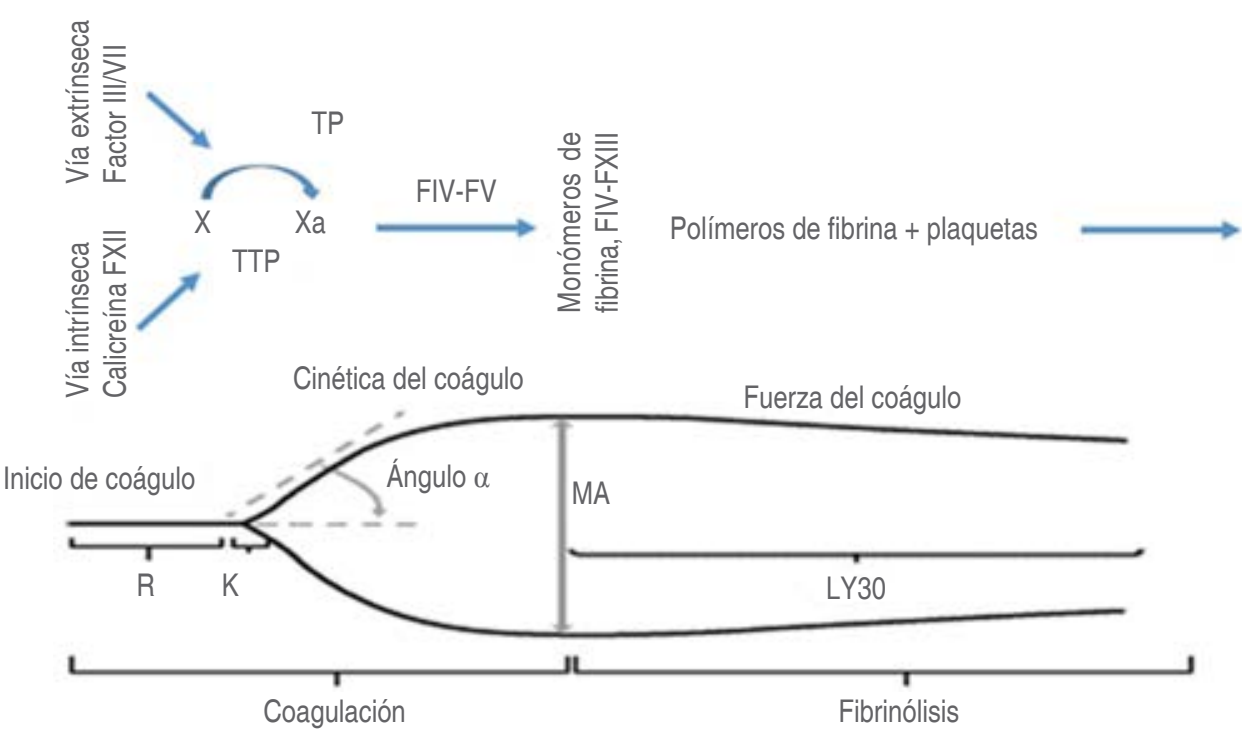

Formación de trombina Parámetro $\mathrm{R}$

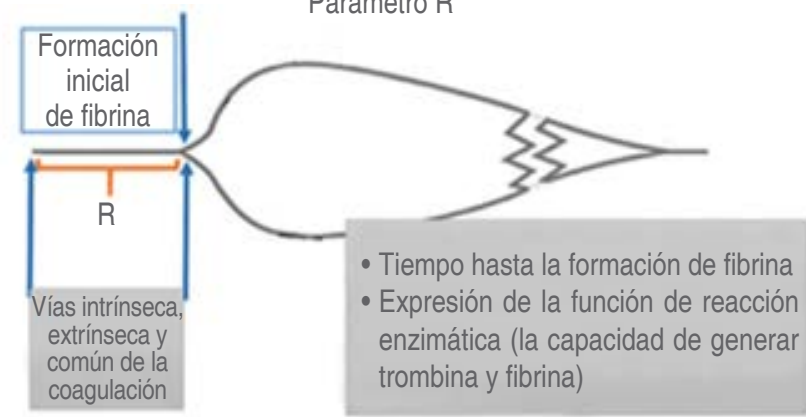

Parámetro $R$ alargado

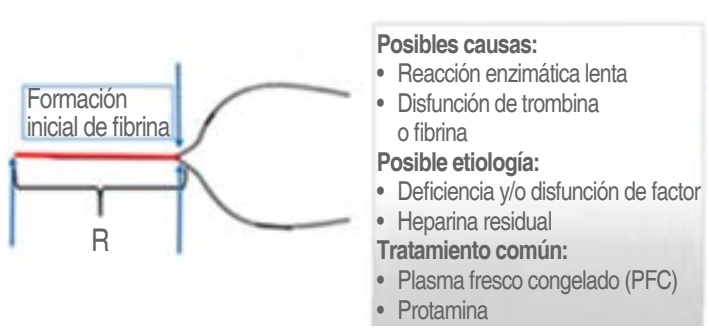

Parámetro R corto

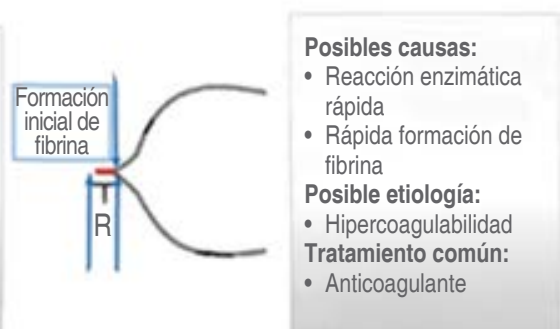

Figura 6:

El parámetro R mide el inicio de
la coagulación, que corresponde
con el tiempo parcial de
tromboplastina (TTP) y tiempo de
tromboplastina (TP) de las pruebas
de laboratorio convencionales.

Los valores aumentados de TP se relacionan con el uso de anticoagulantes, deficiencia de vitamina $\mathrm{K}$, deficiencia de factor VII y problemas en el hígado; también en el caso del uso de suplemento de vitamina K o de píldoras anticonceptivas con estrógeno ${ }^{(1,11,12)}$.

\section{Tiempo de tromboplastina parcial activado (TTPa)}

Este examen también es utilizado para evaluar la hemostasia, sin embargo, permite que se verifique la presencia o ausencia de los factores de coagulación de la vía intrínseca de la cascada de coagulación ${ }^{(11,12)}$.

En este examen, una muestra de plasma sanguíneo es expuesta a reactivos y luego se calcula el tiempo que demora en coagular. En condiciones normales el TTPa es de 21 a 32 segundos. Cuando el TTPa es mayor al tiempo de referencia se sospecha que el paciente utiliza anticoagulantes, como la heparina, o que padece de deficiencia de factores específicos de la vía intrínseca, como los factores XII, XI, IX u VIII los cuales son indicativos de hemofilia ${ }^{(9,11,12)}$. 


\section{Tiempo de trombina (TT)}

El tiempo de trombina es el tiempo en que se forma el coágulo después de la adición de la trombina, la cual ayuda a la conversión del fibrinógeno en fibrina, que garantiza la estabilidad del coágulo(11,12).

Este examen se realiza a partir de la adición de trombina en bajas concentraciones en el plasma sanguíneo. El tiempo de coagulación depende de la cantidad de fibrinógeno presente en el plasma, que normalmente es de $14 \mathrm{a}$ 21 segundos.

El TT está prolongado cuando se utilizan anticoagulantes o tiene deficiencia de fibrinógeno (factor XII) ${ }^{(11,12)}$.

\section{Cuantificación de plaquetas}

La cantidad normal de plaquetas en la sangre es de 150,000 a $450,000 / \mathrm{mm}^{3}$; valores menores al de referencia indican trombocitopenia por probables alteraciones en la médula ósea o infecciones, lo que origina problemas de inicio de la coagulación y aumenta el riesgo de hemorragia masiva ${ }^{(9-11)}$.

\section{Tromboelastografía (TEG)}

El 40\% de los pacientes sometidos a cirugía general, presentan hemorragia, y de ellos de 10 a $12 \%$ no se identifica una causa que justifique el sangrado aun cuando se haya realizado una evaluación preanestésica adecuada y evaluación de pruebas de laboratorio convencionales.

El cálculo para la reposición de elementos formes de la sangre es cualitativo y se reponen a una proporción de 1:1 de concentrados eritrocitarios y plasma. En raras ocasiones se transfunden crioprecipitados o plaquetas. Las unidades hospitalarias que cuentan con los recursos adicionalmente administran antifibrinolíticos. La introducción de la tromboelastografía permite que la trasfusión de derivados sanguíneos sea de mayor calidad y que disminuyan las complicaciones ${ }^{(10)}$.

\section{ASPECTOS TÉCNICOS TROMBOELASTOGRAFÍA (TEG)}

El tromboelastógrafo es un dispositivo computarizado, que mide las propiedades viscoelásticas de la sangre, de una forma dinámica y global, ya que documenta la integración de las plaquetas con la cascada de la coagulación ${ }^{(13,14)}$.

Esta prueba se realiza in vitro, empleando una cubeta o copa en donde se depositan $0.36 \mathrm{~mL}$ de sangre, la cual se gradúa previamente de acuerdo con la temperatura del paciente. La muestra es sometida a un proceso de rotación, la sangre depositada en la copa, se encuentra en contacto con un pin o pasador, el cual está suspendido libremente dentro de la muestra y que, a su vez, está conectado por un extremo a una guía o cable de torsión que lo hace girar; a medida que la sangre coagula y modifica la resistencia del pin; estas

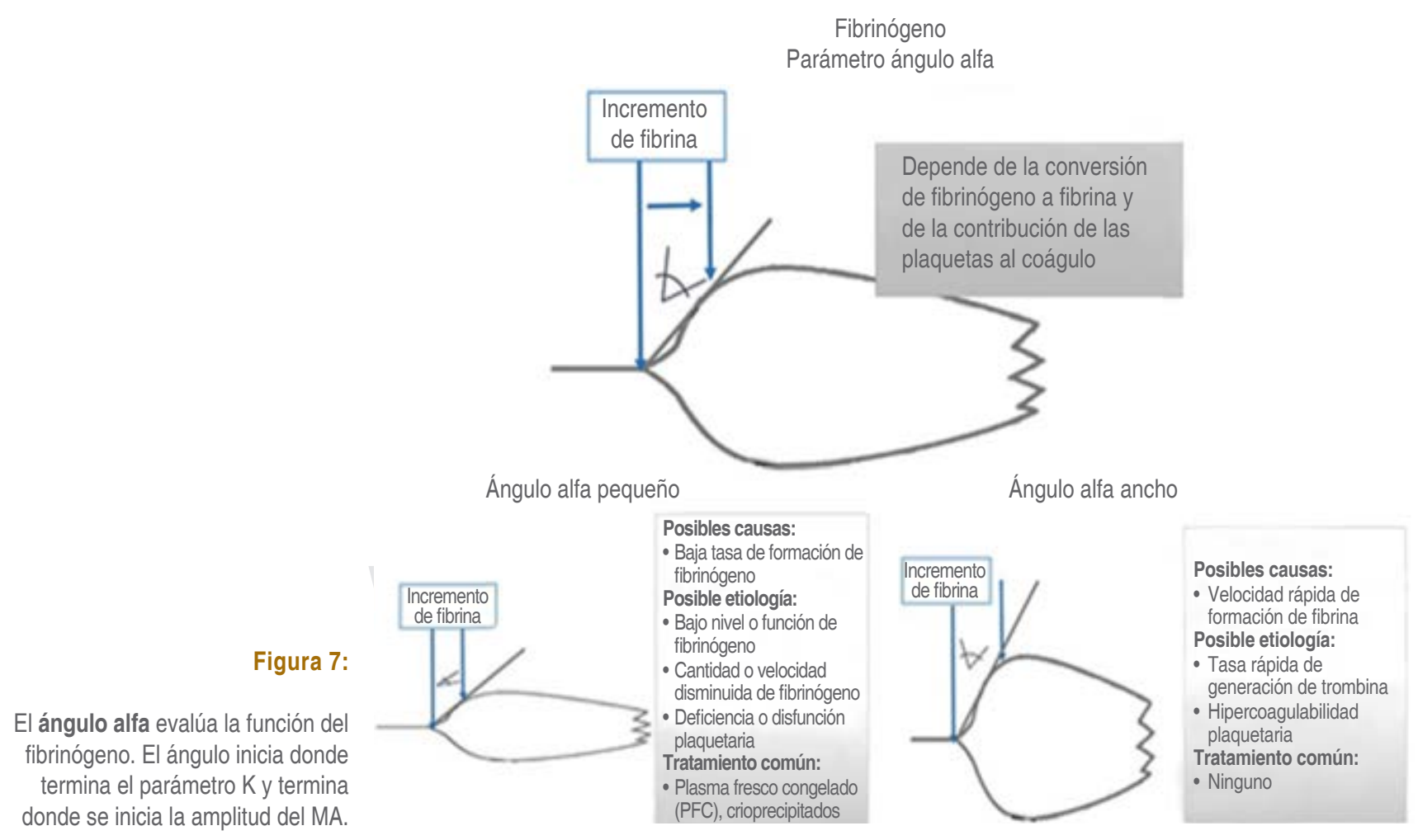




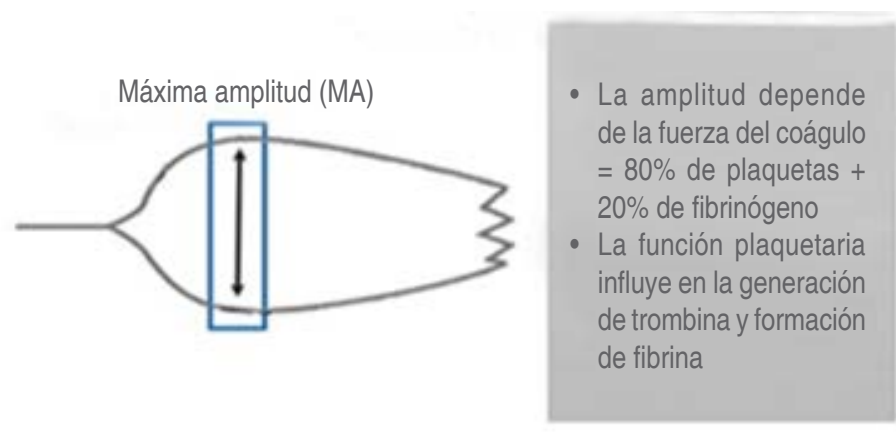

Parámetro MA delgado
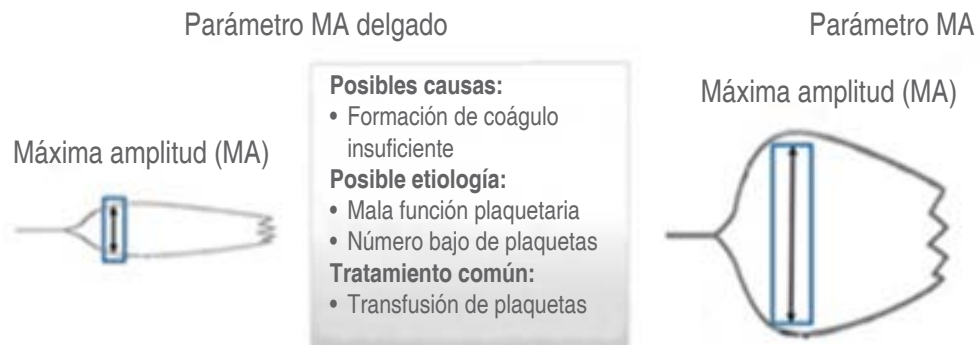

Posibles causas:
- Actividad excesiva
de plaquetas
Posible etiología:
- Hipercoagulabilidad
plaquetaria
Tratamiento común:
- Antiagregantes
plaquetarios

Figura 8:

El parámetro MA (máxima amplitud)
mide la fuerza del coágulo a través
de la función plaquetaria y del
fibrinógeno. Si es amplia es posible
que exista hipercoagulabilidad,
de tener una forma estrecha
indicaría un coágulo insuficiente.

variaciones obtenidas por las características del coágulo y de su etapa evolutiva, son registradas por un transductor electromecánico, el cual, convierte la rotación del pin en una señal eléctrica, graficando así las distintas etapas de la coagulación, es decir, la formación de la fibrina, la retracción del coágulo, la agregación plaquetaria y la lisis del coágulo (Figura 3)(14).

\section{PARÁMETROS DE LA TROMBOELASTOGRAFÍA}

Tiempo de reacción (R): es el tiempo transcurrido desde que se coloca la muestra hasta que empieza la formación de las primeras bandas de fibrina. Puede prolongarse cuando existen deficiencias de factores de la coagulación, como la acción de la heparina, warfarina o las heparinas de bajo peso molecular. Su acortamiento implica hipercoagulabilidad de cualquier etiología y representa la funcionalidad de los factores de coagulación ${ }^{(7,15,16)}$.

Constante de coagulación (K): tiempo que transcurre desde el comienzo de la formación de fibrina hasta que el coágulo alcance $20 \mathrm{~mm}$, es decir, evalúa el tiempo de formación del coágulo ${ }^{(14,15)}$.

Ángulo alfa: es la velocidad de formación del coágulo. La angulación aumenta cuando existe hiperagregabilidad plaquetaria o elevación del fibrinógeno, y disminuye en presencia de agentes anticoagulantes o de antiagregantes plaquetarios, representa principalmente la funcionalidad del fibrinógeno $^{(13-15)}$.
Amplitud máxima (MA): se mide en milímetros y evalúa el momento en que el coágulo alcanza su máxima fuerza, representa principalmente la función plaquetaria ${ }^{(14-16)}$.

LY30: es la medida en porcentaje de la lisis del coágulo después de que ha alcanzado la máxima amplitud, reflejando la estabilidad del coágulo. Por lo tanto, se aumenta en procesos en los cuales exista fibrinólisis ${ }^{(14,15)}$ (Figura 4).

Dentro de las principales ventajas que ofrece la TEG es el análisis in vitro en la relación entre los diferentes componentes de la coagulación; es así como se puede observar la relación existente entre plaquetas, fibrinógeno y proteínas de la coagulación de forma integral. El análisis de los tiempos de coagulación se realiza de forma aislada en el plasma del paciente y no hay forma de observar su asociación con el entorno fisiológico del mismo ${ }^{(13,15)}$.

En la TEG, las mediciones del TP, TTPa y TT están representadas en la medición del R y K. La función de las plaquetas se puede observar en MA, así como el inicio de la lisis del coágulo (Figura 5).

La tromboelastografía se ha utilizado con mayor frecuencia en cirugías en las que se espera un sangrado mayor al 40\% del volumen sanguíneo circulante de un paciente, que generalmente son cirugías cardíacas, de trasplante de hígado o de malformaciones arteriovenosas; sin embargo, ésta es una herramienta muy útil en otro tipo de cirugías, ya que ofrece información útil para determinar si es necesario el tratamiento de transfusión y en caso de ser necesario, permite identificar de manera especí- 
fica el tipo de tratamiento necesario. De ahí la importancia de conocer e interpretar adecuadamente los elementos de la tromboelastografía, que permita integrar las pruebas de coagulación convencionales con la función plaquetaria y así dar una idea más global de la fisiología de la hemostasia.

En pacientes con alteraciones de la coagulación, con los patrones de la tromboelastografía, es posible identificar con precisión el defecto subyacente de la cascada de coagulación, lo que permite tener una idea clara de en qué fase se encuentra alterada y, de esta manera, facilitar la decisión sobre el tratamiento que requiere el paciente ${ }^{(17)}$ (Figuras 6 a 10).

Shore-Lesserson y colaboradores demostraron que el uso rutinario del TEG implica menos transfusión de componentes sanguíneos al compararse con una terapia transfusional, basada en pruebas de laboratorio rutinarias en pacientes llevados a cirugía cardiovascular mayor ${ }^{(17)}$. Esto implica una

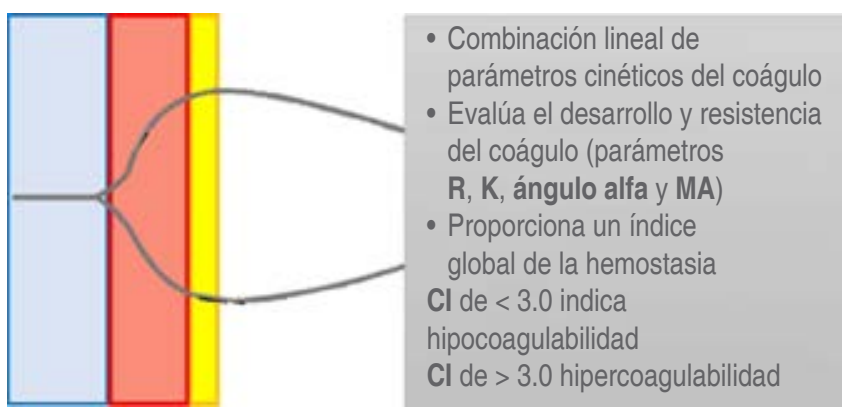

Figura 9: El índice de coagulación $(\mathrm{Cl})$ mide la calidad del coágulo, con base en los parámetros $\mathrm{R}$, K, ángulo alfa y MA (amplitud máxima). Si algunos de los parámetros cambian modifican el resultado del $\mathrm{Cl}$. disminución de los costos y de la exposición innecesaria por parte de los pacientes a la sangre y sus derivados.

\section{Ventajas y desventajas de la tromboelastografía}

\section{Ventajas:}

1. Proceso sencillo y de fácil interpretación.

2. Requiere mínima cantidad de sangre $(0.36 \mathrm{~mL})$.

3. Evalúa de forma global la formación del coágulo hasta su destrucción.

4. Ayuda a diferenciar entre sangrado debido a alteraciones de la coagulación y a hemostasia quirúrgica inadecuada.

5. Detecta estados de hipercoagulabilidad, especialmente en trauma y en cirugía, además sirve como predictor de eventos trombóticos.

6. Racionaliza la utilización de productos sanguíneos y hemostáticos.

7. Se pueden realizar como parte de la evaluación preanestésica.

\section{Desventajas:}

No identifica:

1. Factores de coagulación: VII, IX, X.

2. Inhibidores: antitrombina, proteína $C$, proteína $S$.

3. Activadores: tromboxanos A-2, ADP.

Es importante mencionar que las pruebas de coagulación convencionales TP, TTPa, TT tampoco identifican los factores de coagulación VII, IX y X, los inhibidores anti-

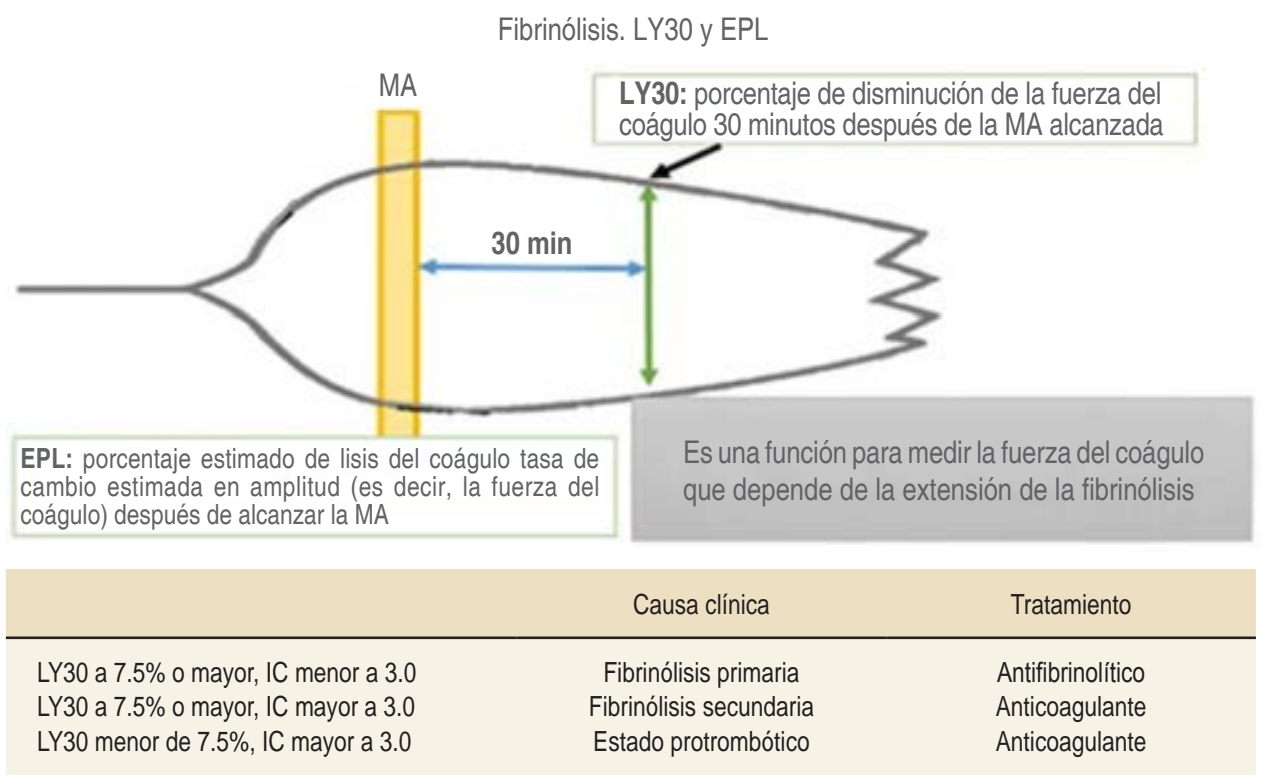

Figura 10:

Representación de parámetros LY30 y porcentaje estimado de lisis del coágulo de la tromboelastografía. De resultar elevados se requerirá de antifibrinolítico, y de ser menor al valor normal se tratarán con anticoagulantes. 

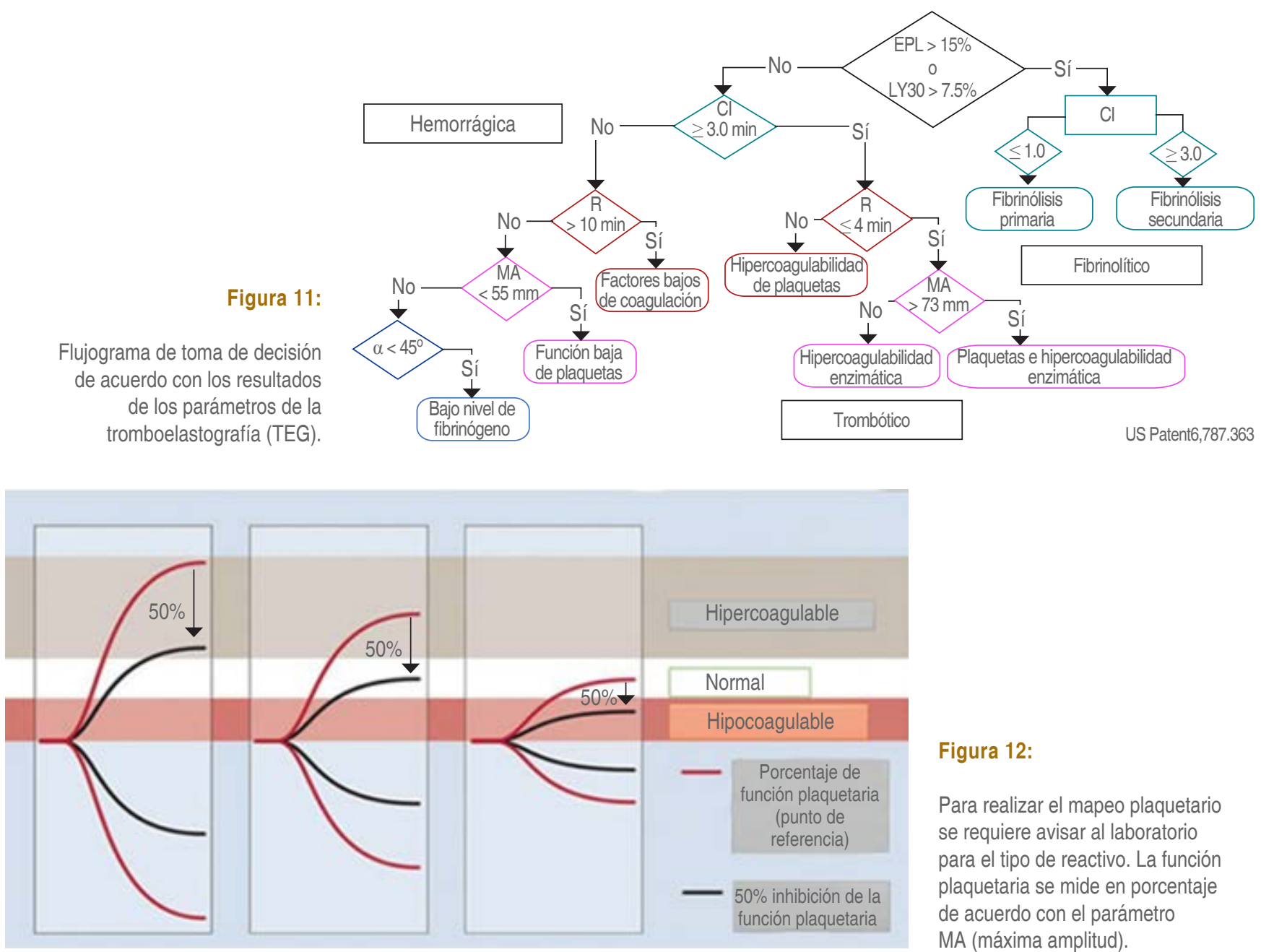

Figura 12:

Para realizar el mapeo plaquetario se requiere avisar al laboratorio para el tipo de reactivo. La función plaquetaria se mide en porcentaje de acuerdo con el parámetro MA (máxima amplitud).

trombina, proteína C, proteína $\mathrm{S}$, así como activadores de tromboxanos A-2 y ADP. Lo que significa que la TEG no presenta desventajas adicionales a las pruebas de coagulación convencionales.

La utilidad de la TEG no sólo se limita a la toma de decisiones en cirugías con sangrado, sino que también confiere una mayor seguridad en la toma de decisiones preoperatorias a los pacientes que toman antiagregantes plaquetarios de manera habitual, a través del mapeo plaquetario (Figura 11)(18).

\section{MAPEO PLAQUETARIO}

Muchos protocolos quirúrgicos suspenden la administración de inhibidores plaquetarios como lo son el clopidogrel o el ácido acetilsalicílico antes de una cirugía para minimizar el riesgo de sangrado, pero ¿qué pasa si interrumpimos una medicación antiplaquetaria en un paciente que está predispuesto a un evento trombótico? Al facilitar o inhibir la función plaquetaria antes de la cirugía sin entender el estado basal del paciente podríamos ponerlo en riesgo de sufrir una hemorragia o una trombosis e incrementar el costo de su cuidado perioperatorio. Si se le suministra una dosis demasiado baja de antiagregante plaquetario postoperatoria podría ocurrir una trombosis, mientras que si se le suministra demasiada inhibición podría presentarse sangrado. El ensayo del mapeo plaquetario (Platelet Mapping ${ }^{\circledR}$ Assay) mide la función plaquetaria y muestra el nivel de inhibición de la agregación plaquetaria del paciente y el riesgo trombótico o hemorrágico. Evaluando el efecto de las drogas administradas para sus diferentes tratamientos, la medición del mapeo plaquetario se logra a través del ADP o ácido araquidónico modificando la medición del MA e inhibiendo los efectos de los agentes antiplaquetarios, los resultados se presentan en una interpretación de porcentaje de inhibición ${ }^{(19)}$ (Figura 12).

La TEG es una herramienta que ayuda a la decisión del tratamiento del sangrado perioperatorio; sin embargo, existen otros elementos a considerar: 
1. Coagulopatía dilucional. Se presenta tras la administración de grandes cantidades de volumen (coloides, cristaloides) intravenoso en la reanimación inicial para mantener la volemia ${ }^{(20)}$.

2. Hipotermia. Provoca disfunción plaquetaria severa y bloqueo enzimático de las reacciones fisiológicas de la coagulación $^{(21)}$.

3. Politransfusión. La transfusión abundante de sangre almacenada conlleva coagulopatía ${ }^{(22,23)}$. Siempre que el aporte de hemoderivados sea abundante hay que considerar las alteraciones debidas al almacenamiento de los hemocomponentes, que está dada por:
a. Dificultad en la entrega de oxígeno a los tejidos.
b. Hipotermia.
c. Intoxicación por citrato: disminución de calcio.
d. Alteraciones electrolíticas.
e. Acidosis.
f. Hiperglucemia.

4. Acidosis. El valor del pH sanguíneo es el factor pronóstico más importante de coagulopatía, con una disminución en la actividad del FVIIa, del complejo FT/FVIIa y del complejo $\mathrm{Xa} / \mathrm{Va}$ cuando el $\mathrm{pH}$ se aproximaba a valores de 7.0, la actividad enzimática de los factores de coagulación se encuentra disminuida hasta en $90 \%{ }^{(23)}$.
La tromboelastografía es una herramienta útil para evaluar y seleccionar el tratamiento adecuado para los pacientes quirúrgicos que presentan algún trastorno de la coagulación y que derivan en un sangrado mayor. El análisis de costo-beneficio del uso de la TEG es favorable en todos los casos, ya que de requerirse transfusión de derivados sanguíneos, la información que proporciona la TEG garantiza que el tipo y la calidad de trasfusión serán óptimos para el paciente, puesto que minimizan los riesgos de politransfusión. El uso de la TEG debería ser rutinario, ya que permitiría mejorar los alcances pronósticos de la evaluación preanestésica y durante la presencia de sangrado transquirúrgico y su realización.

La Figura 13 ejemplifica una paciente con un estado de anticoagulación representado por una prolongación en el parámetro $\mathrm{R}$, por lo que su tratamiento debe ser con plasma fresco congelado. La Figura 14 es una tromboelastografía de un paciente con estado de hipercoagulabilidad por consumo, porque todos los parámetros de la misma están modificados y la campana es ancha y su tratamiento debe ser con antifibrinolíticos.

\section{CONCLUSIÓN}

La inclusión rutinaria del TEG en la evaluación preanestésica mejora los alcances pronósticos de esta evaluación, ya que da información global del estado de coagulación del paciente que será sometido a cualquier cirugía, y con ayuda del mapeo

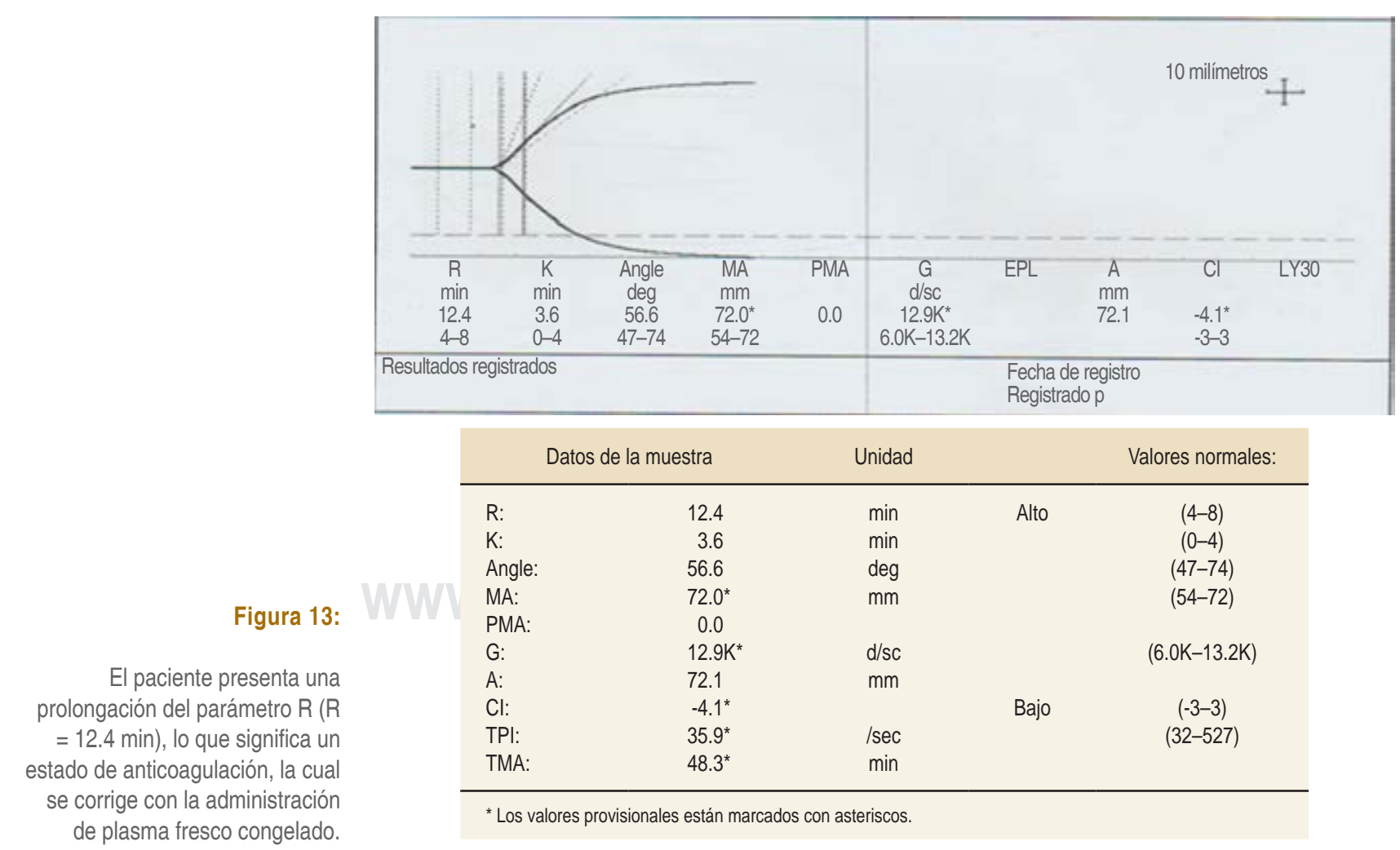




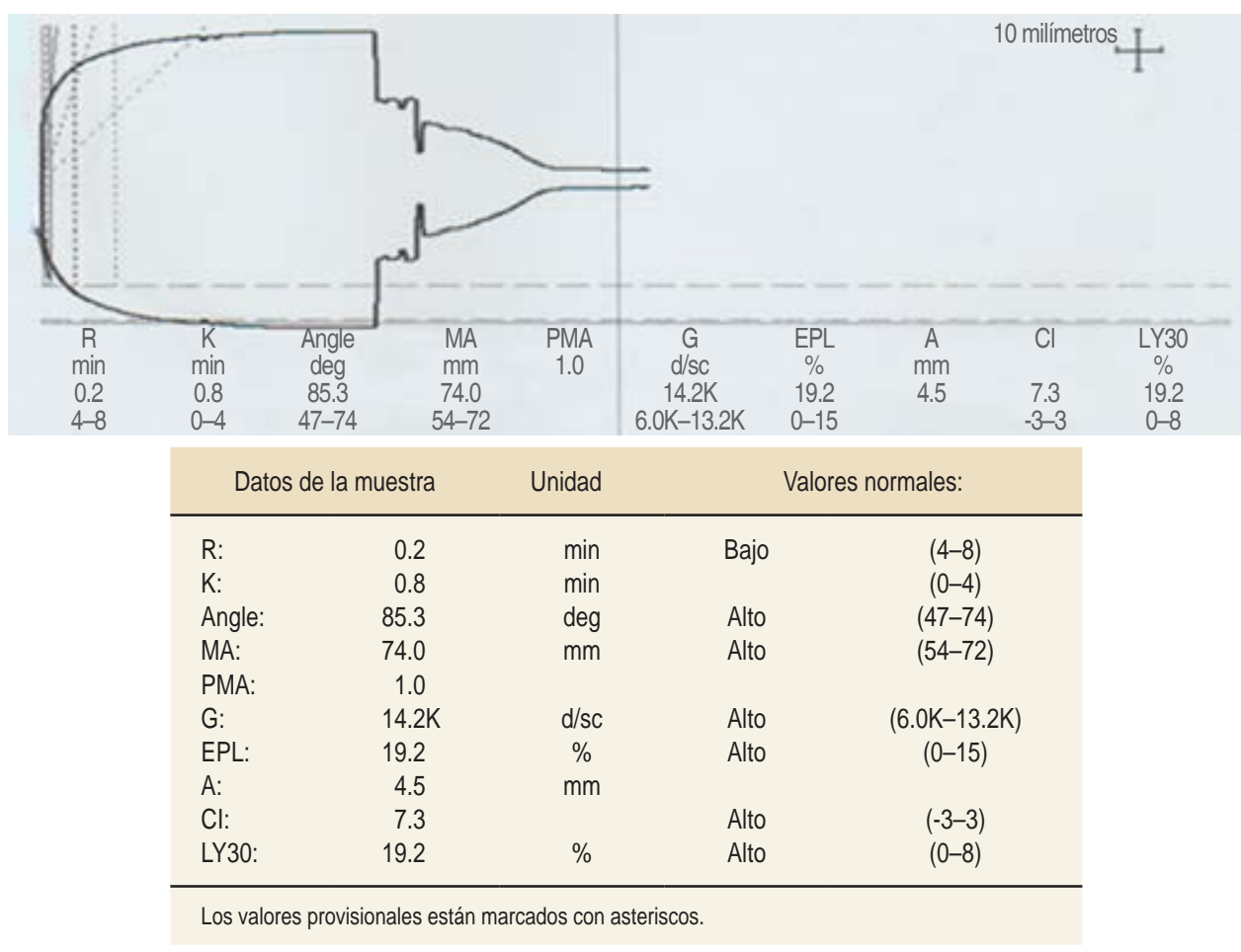

Figura 14:

El paciente presenta hipercoagulabilidad por consumo, por lo que el tratamiento debe ser con antifibrinolíticos. plaquetario puede auxiliar a la decisión de suspender la administración de antiagregantes plaquetarios como ácido acetilsalicílico o clopidogrel.

$\mathrm{Al}$ ser la TEG un análisis rápido, que ofrece resultados en los primeros 15 minutos, se convierte en una herramienta útil en el período transquirúrgico, ya que se puede tomar la muestra de sangre del paciente $(0.36 \mathrm{~mL})$ en el momento que inicia el sangrado y de acuerdo con su resultado puede diferenciar si el sangrado es debido a alteraciones de la coagulación o a una hemostasia quirúrgica inadecuada, y así seleccionar el tratamiento apropiado para los pacientes con sangrado mayor.

El uso de la TEG como herramienta pre- y transanestésica no incrementa de manera significativa los costos de la cirugía y minimiza los riesgos de ésta, por lo que el análisis costo-beneficio es favorable al uso rutinario de la TEG, el cual se verá reflejado en un incremento de la calidad de la atención al paciente.

\section{REFERENCIAS}

1. Chuquimia Zarate LD, Flores Pilco YMR. Hemostasia coagulación y transfusión sanguínea en cirugía. Rev. Act Clin Med. 2011;15:876-879.

2. Versteeg HH, Heemskerk JW, Levi M, Reitsma PH. New fundamentals in hemostasis. Physiol Rev. 2013;93:327-358.

3. Yan Y, Xu LC, Vogler EA, Siedlecki CA. Contact activation by the intrinsic pathway of blood plasma coagulation. In: Siedlecki CA. Hemocompatibility of biomaterials for clinical applications: bloodbiomaterials interactions. Oxford, UK: Woodhead Publishing; 2018. pp. 3-28.

4. Ceresetto JM. Global interpretation of coagulation tests. Hematología. 2017;21:69-76.

5. Pant A, Kopec AK, Luyendyk JP. Role of the blood coagulation cascade in hepatic fibrosis. Am J Physiol Gastrointest Liver Physiol. 2018;315:G171-G176.

6. Guerrero B, López M. Generalidades del sistema de la coagulación y pruebas para su estudio. Invest Clín. 2015;56:432-454.

7. Galvez K, Cortes C. Tromboelastografía: nuevos conceptos en la fisiología. Rev Colomb Anestesiol. 2012;40:224-230.

8. Cervera Bravo A. Fisiopatología y trastornos de la coagulación hereditarios más frecuentes. Pediatr Integral. 2012;16:387-398.
9. Maegele M, Schochl H, Cohen MJ. An update on the coagulopathy of trauma. Shock. 2014;41 Suppl 1:21-25.

10. López-Santiago N. Pruebas de coagulación. Acta Pediatr Mex. 2016;37:241-245.

11. Zimring JC. Introduction to coagulation testing. In: Hillyer CD, Shaz BH, Zimring JC, Abshire TC, editors. Transfusion medicine and hemostasis, clinical and laboratory aspects. New York, USA: Elsevier; 2009. pp. 601-605.

12. Flores-Rivera OI, Ramírez-Morales K, Meza-Márquez JM et al. Fisiología de la coagulación. Rev Mex Anest. 2014;37:382-386.

13. Macafee B, Campbell JP, Ashpole K, Cox M, Matthey F, Acton L, et al. Reference ranges for thromboelastography (TEG( $\left({ }^{\circledR}\right)$ ) and traditional coagulation tests in term parturients undergoing caesarean section under spinal anaesthesia. Anaesthesia. 2012;67:741-747.

14. Wikkelso A, Wetterslev J, Moller AM, Afshari A. Thromboelastography (TEG) or rotational thromboelastometry (ROTEM) to monitor haemostatic treatment in bleeding patients: a systematic review with meta-analysis and trial sequential analysis. Anaesthesia. 2017;72:519-531.

15. Pérez-Calatayud AA, Giraldo-Cadavid LF, Aguilar-Vidales K, LozaGallardo LR, Escobar-Herrera G, González-Hernández J, et al. Informe 
del primer caso de manejo transfusional guiado por tromboelastometría rotacional en México y revisión de la bibliografía . Cir Cir. 2019;87:1-7.

16. Barraza-Cervantes AJ, Díaz-Franco SD, Sosa-García JO. Tromboelastografía como guía para la toma de decisiones en el perioperatorio. Rev Mex Anest. 2015;38:277-284.

17. Shore-Lesserson L, Manspeizer HE, DePerio M, Francis S, Vela-Cantos F, Ergin MA. Thromboelastography-guided transfusion algorithm reduces transfusions in complex cardiac surgery. Anesth Analg. 1999;88:312-319.

18. Franceschi JL. Tromboelastografía y sus aplicaciones clínicas. Rev Med Panama. 2015;35:30-35.

19. Weitzel NS, Weitzel LB, Epperson LE, Karimpour-Ford A, Tran ZV, Seres T. Platelet mapping as part of modified thromboelastography (TEG®) in patients undergoing cardiac surgery and cardiopulmonary bypass. Anaesthesia. 2012;67:1158-1165.
20. Cruz-Guerrero RF, Jiménez-Ávila JM, González-Cisneros AC, Rivera-Villa AH, Sánchez-Chávez FA, Rincón-Gómez MR, et al. Almidón como factor de riesgo para el incremento del sangrado transquirúrgico en la artroplastia total de cadera. Cir Cir. 2019;87:164-169.

21. Crisóstomo-Pineda MM, Hernández-Pérez AL, Ordóñez-Espinosa G, Riera-Kinkel C. La hipotermia y sus efectos durante la anestesia en niños. Rev Mex Pediatr. 2011;78:131-138.

22. Sherrod BA, Baker DK, Gilbert SR. Blood transfusion incidence, risk factors, and associated complications in surgical treatment of hip dysplasia. J Pediatr Orthop. 2018;38:208-216.

23. Maldonado Rojas M, Piña Farías L, Vásquez Rojas M, Toro Opazo C. Complicaciones asociadas a la transfusión masiva. Rev Cubana Hematol Inmunol Hemoter. 2013;29:46-61. 